\title{
An unfortunate case of post-ERCP complications
}

\author{
Massimo Fiorini $^{1} \cdot$ Antonello Pietrangelo $^{1} \cdot$ Alberto Vegetti $^{1}$
}

Received: 9 May 2016/Accepted: 20 May 2016/Published online: 10 June 2016

(C) SIMI 2016

A 47-year-old woman was admitted to our internal medicine unit with a 1-week history of recurrent abdominal pain, without nausea, vomit, fever or itching. Physical examination revealed a positive Murphy's sign, and laboratory tests were compatible with cholestasis and mild hepatitis (total bilirubin $1.24 \mathrm{mg} / \mathrm{dL}$, direct bilirubin $0.5 \mathrm{mg} / \mathrm{dL})$, AST $103 \mathrm{U} / \mathrm{L}$, ALT $231 \mathrm{U} / \mathrm{L}, \mathrm{Y}-\mathrm{GT} 635 \mathrm{U} / \mathrm{L}$, alkaline phosphatase $269 \mathrm{U} / \mathrm{L}$, bile acids $10.9 \mu \mathrm{mol} / \mathrm{L}$, amylase $64 \mathrm{U} / \mathrm{L}$, lipase $33 \mathrm{U} / \mathrm{L}$.

Abdominal ultrasound showed a dilatation of the intrahepatic/extrahepatic bile ducts and gallbladder sludge. She was diagnosed with three small common bile duct stones by magnetic resonance cholangiopancreatography (Fig. 1a, b): an endoscopic biliary sphincterotomy was performed via ERCP, and the stones were successfully extracted with a Fogarty catheter without apparent complications. A nasobiliary tube (NBT) was left in place to allow future controls and in preparation for a cholecystectomy.

Eight hours post-procedure, the patient suddenly presented a severe upper quadrant abdominal pain, without tenderness or signs of peritonitis, with intermittent fever (up to $39{ }^{\circ} \mathrm{C}$ ) and chills. There were no signs of hemodynamic instability. Serum biochemistry demonstrated a rise of pancreatic enzymes and inflammatory markers: amylase $1395 \mathrm{U} / \mathrm{L}$, lipase $2837 \mathrm{U} / \mathrm{L}$, white blood cells $10.99 \times 10^{9} /$ $\mathrm{L}$, serum C-reactive protein $1.3 \mathrm{mg} / \mathrm{dL}$. Samples for blood cultures were collected. Suspecting an infected acute necrotizing hemorrhagic pancreatitis, total parenteral

Massimo Fiorini

massimo.fiorini.88@ hotmail.it

1 Internal Medicine 2, Center for Hemochromatosis, University Hospital of Modena, Via del Pozzo 71, Modena, MO, Italy nutrition regimen was started, and intravenous ciprofloxacin was administered together with omeprazole.

On day 1 , despite the treatment, abdominal pain worsened so intravenous paracetamol and tramadol were dispensed as analgesics providing only poor pain control. A computed tomography (CT) was performed: acute interstitial edematous pancreatitis and a subcapsular hepatic lesion $(\varnothing 4.5 \mathrm{~cm})$ in the second liver segment, which, according to the radiologist, was compatible with a biloma (Fig. 1c, d), were documented.

On day 2, amylase and lipase levels rapidly declined, which suggested recovery from the acute pancreatitis, a common complication of ERCP. However, fever with chills and particularly abdominal pain persisted even after $48 \mathrm{~h}$ of treatment with ciprofloxacin. There was an increase of inflammatory markers (white blood cells $17.78 \times 10^{9} / \mathrm{L}$, serum C-reactive protein $24.4 \mathrm{mg} / \mathrm{dL}$ ). Blood and urine culture samples were persistently negative, as chest X-ray study, serum $\beta$-D-glucan and galactomannan assessment. The patient underwent a second (piperacillin/tazobactam) and a third line (meropenem) broad-spectrum antibiotic therapy, without benefit. Therefore, on day 6, a trans-cutaneous drainage under ultrasound guidance of the hepatic lesion was planned: neither bile nor purulent material were drained, but only a small amount of sterile clotted blood, indicating a subcapsular hepatic hematoma. On the same day the NBT placed during ERCP was removed. The subsequent NBT tip microbiological assays revealed a colonization by Candida glabrata, Candida albicans and methicillin-resistant Staphylococcus aureus. An antibiogram/antimicogram-guided therapy with teicoplanin and fluconazole was started: defervescence finally occurred within $48 \mathrm{~h}$. The observed clinical evolution suggested that a second most common complication of ERCP, cholangitis, was successfully treated. Yet, abdominal pain persisted in 
Fig. 1 a, b Evidence of common bile duct stones by magnetic resonance

cholangiopancreatography. c, d Subcapsular hepatic lesion (hematoma) in the second liver segment by CT-scan
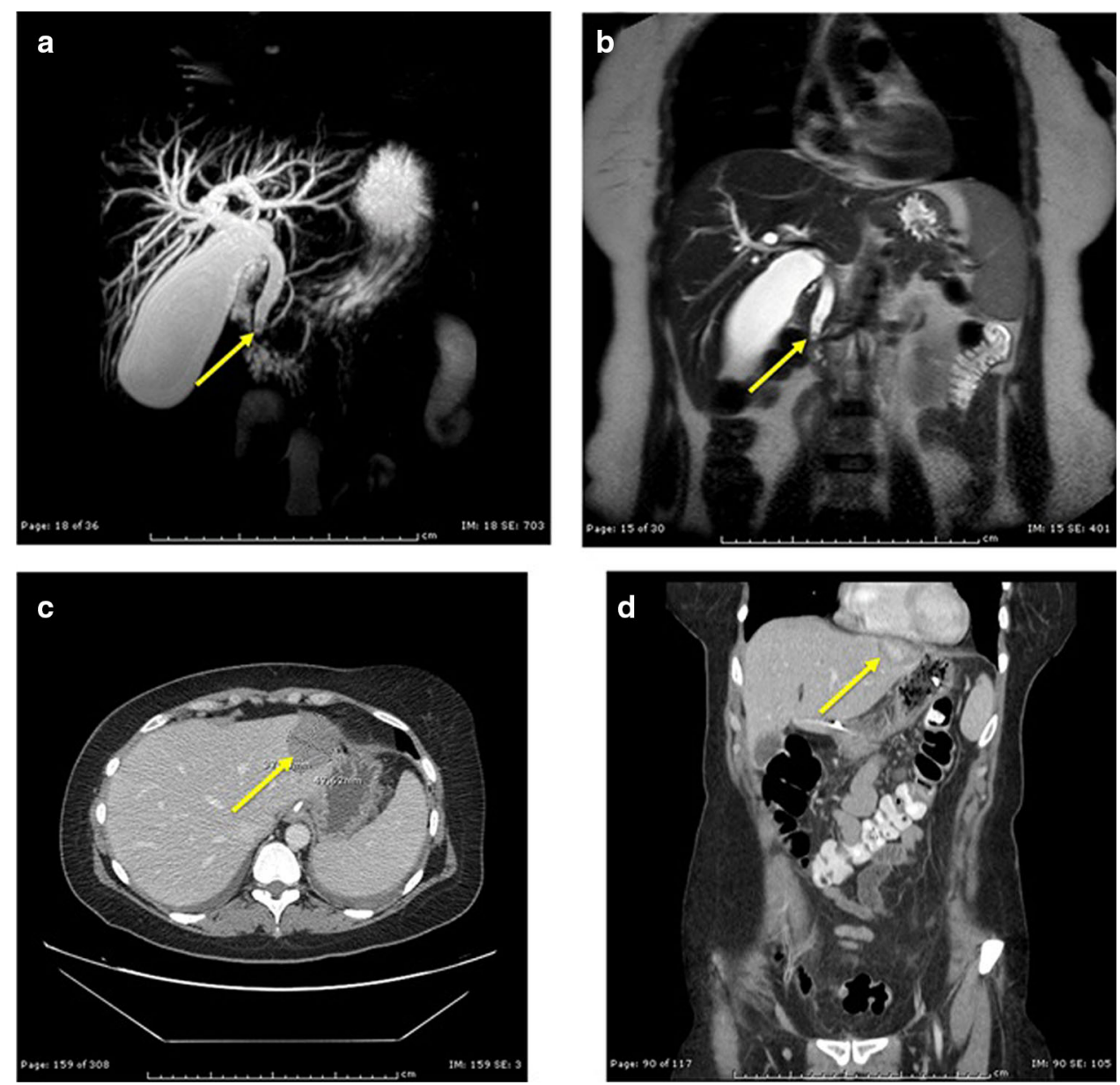

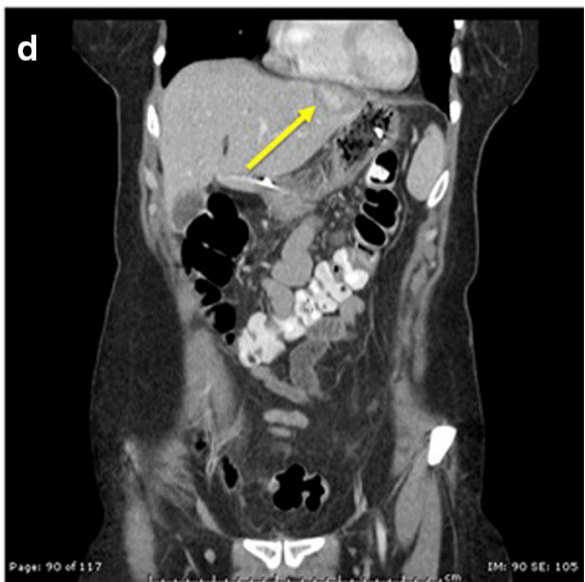

spite of the recovery from infection. On the following days a gradual resorption of the hematoma was eventually demonstrated by ultrasound evaluation, until its complete resolution. As a result, abdominal pain gradually improved and finally disappeared. Refeeding was started without any complication. The patient was discharged, and after 1 month underwent a successfully cholecystectomy.

Endoscopic retrograde cholangiopancreatography (ERCP) is one of the least invasive and most frequently performed procedures in the diagnosis and treatment of biliary-pancreatic diseases. Severe post-ERCP complications occur in $2.5-8 \%$ of cases, with a mortality rate of $0.5-1 \%$ [1]. The most common are acute pancreatitis, cholangitis, perforation and bleeding due to sphincterotomy [1-3]. Subcapsular hepatic hematoma is a rare complication described by few reports [1, 3, 4]. Its low incidence could also be affected by the habit of not performing post-procedural radiological controls [3]. Subcapsular hepatic hematoma may originate from guide wire drilling of the biliary tree and the subsequent rupture of a small caliber vessel during ERCP [1, 3, 4]. Appearance of post-ERCP abdominal pain or hypotension are the triggering signs that should suggest the possibility of a subcapsular hepatic hematoma $[1,3]$. In the majority of patients, the treatment is conservative. If the hematoma is refueled by an active bleeding vessel, embolization of the vessel is indicated. A surgical approach instead, should be considered in case of rapid worsening of the clinical condition (hemodynamic instability, intra-abdominal bleeding, peritonitis) [1]. Due to the risk of infection, there is general consensus to administer prophylactic antibiotic therapy [4].

In this case, according to internal hospital procedures, a pre-cholecystectomy ERCP was chosen instead of surgery in the first place. In 2013, a Cochrane review reported no significant difference in the mortality and overall morbidity rates between laparoscopic common bile duct exploration and the pre-, intra- and postoperative ERCP [5]. Yet, the authors were not able to assess the impact of the procedures' specific morbidity such as pancreatitis, cholangitis and bleeding, an issue that remains undefined.

After the endoscopic procedure, the patient developed simultaneously three post-ERCP complications: two common (acute interstitial edematous pancreatitis and cholangitis) and one rare (subcapsular hepatic hematoma). The sudden and severe abdominal pain appearing after ERCP was initially ascribed to acute pancreatitis and cholangitis, 
which may have well contributed at early stages. However, once those conditions were successfully managed, the pain persisted and even worsened, to finally resolve only when the hematoma gradually disappeared.

The NBT was probably contaminated during tube positioning and/or ERCP. In fact, C. glabrata, C. albicans and methicillin-resistant $S$. aureus can colonize nasal, pharyngeal and small intestinal mucosa. Thus the presence of a colonized NBT sustained a local biliary infection that resolved upon removal of a source of infection (i.e., NBT) and targeted antibiotic treatment.

\section{Compliance with ethical standards}

\section{Conflict of interest None.}

Statement of human and animal rights This article does not contain any studies with human participants or animals performed by any of the authors.
Informed consent The patient, who is not identifiable in this paper, gave us consent to deal with her case.

\section{References}

1. Fei BY, Li CH (2013) Subcapsular hepatic haematoma after endoscopic retrograde cholangiopancreatography: an unusual case. World J Gastroenterol 19:1502-1504

2. Committee ASoP, Anderson MA, Fisher L et al (2012) Complications of ERCP. Gastrointest Endosc 75:467-473

3. Orellana F, Irarrazaval J, Galindo J et al (2012) Subcapsular hepatic hematoma post ERCP: a rare or an underdiagnosed complication? Endoscopy 44(Suppl 2 UCTN):E108-E109

4. Del Pozo D, Moral I, Poves E et al (2011) Subcapsular hepatic hematoma following ERCP: case report and review. Endoscopy 43(Suppl 2 UCTN):E164-E165

5. Dasari BV, Tan CJ, Gurusamy KS et al (2013) Surgical versus endoscopic treatment of bile duct stones. Cochrane Database Syst Rev 12:CD003327 